(C) 1999 International Press

Adv. Theor. Math. Phys. 3 (1999) 249-278

\title{
On the Generalized Casson Invariant
}

\author{
George Thompson
}

\author{
ICTP \\ P.O. Box 586 \\ 34100 Trieste \\ Italy \\ thompson@ictp.trieste.it
}

\begin{abstract}
The path integral generalization of the Casson invariant as developed by Rozansky and Witten is investigated. The path integral for various three manifolds is explicitly evaluated. A new class of topological observables is introduced that may allow for more effective invariants. Finally it is shown how the dimensional reduction of these theories corresponds to a generalization of the topological $\mathbf{B}$ sigma model.
\end{abstract}

e-print archive: http://xxx.lanl.gov/abs/hep-th/9811199 


\section{Introduction}

The Casson invariant, a three manifold invariant, has been with us since 1985. Originally it was defined, by Casson, for $\mathbb{Z} H S$ 's (integral homology spheres) [6]. In this situation, Taubes showed that the Casson invariant can be viewed as the Euler characteristic of the Floer homology of flat $S U(2)$ connections on the integral homology sphere [19]. There is a path integral representation of the invariant, due to Witten, which formally gives back the construction of Taubes [21]. Atiyah and Jeffrey interpreted this result as being a definition of a 'regularised' Euler characteristic of the (infinite dimensional) space of $S U(2)$ connections on $\mathbb{Z H S}$ 's. An alternative interpretation of the path integral was given in [2]. There it was argued that the Casson invariant is the Euler Characteristic of the moduli space of flat connections (when the space is disjoint it is the Euler characteristic of each component sumed with signs given by spectral flow) ${ }^{1}$. This interpretation is in keeping with the work of Taubes.

The invariant was generalised to rational homology spheres by Walker in [20]. After much activity, Lescop gave a surgery formula for the invariant so that it could be extended to all three manifolds, see [12] and references therein. These generalisations are for the $S U(2)$ invariant. There is now an $S U(3)$ extension of the Casson invariant for $\mathbb{Z H S}$ 's due to Boden and Herald [5]. Each generalisation is confronted by various analytical problems which had to be overcome. The path integral version makes sense, as it stands, for any three manifold and for any gauge group and so, in principal, offers a handle on the Casson invariant that goes beyond what is mathematically accesable at present. Unfortunately the path integral in question proved very difficult to evaluate.

The situation changed dramatically as a consequence of the solution of the $N=2$ super Yang-Mills theory in four dimensions [17]. This work had profound consequences for the study of four manifold invariants. On passing to three dimensions ${ }^{2}$ Seiberg and Witten [18] gave a solution to the $N=4$ super Yang-Mills theory with gauge group $S U(2)$ in the coulomb branch. The moduli space of the theory was conjectured to be the Atiyah-Hitchin two monopole moduli space. To pass to the path integral representation of the Casson invariant one starts with the physical theory and one twists it (I

\footnotetext{
${ }^{1} \mathrm{~A}$ description of this construction which is perhaps more accessible to mathematicians can be found in a forthcoming book [14]. There one will also find a discussion on the three dimensional analogue of the Seiberg-Witten invariant and its relationship to the Casson invariant.

${ }^{2}$ In section 5 I explain, rather broadly, how one passes from a higher dimensional manifold to a lower dimensional one.
} 
describe this in the body of the paper). Since the topological theory ought not to depend on which scale we are looking at, twisting the full theory or twisting the low energy effective theory should yield the same invariant. The Casson invariant is therefore given by a particular path integral part of whose data includes the integration over the space of maps from the three manifold to the Atiyah-Hitchin space $X_{\mathrm{A} H}$.

The Rozansky-Witten invariant $Z_{X}^{R W}[M]$ is then one manifestation of the path integral invariant [16], the manifestation in which one has a supersymmetric sigma model of maps from $M$ to some hyper-Kähler manold $X$. This corresponds to the Casson invariant of $M$ when $X=X_{\mathrm{A} H}$. Rozansky and Witten establish that in this case one does indeed reproduce all the known general results of the Casson invariant including the surgery formula of Lescop [12]. One can read this sucess in the opposite direction, namely that this confirms the conjecture of Seiberg and Witten that for the physical theory the coulomb branch moduli space is $X_{\mathrm{A} H}$.

Some immediate consequences of [16] are that for $b_{1}(M)>3$ the invariant vanishes and for $1 \leq b_{1}(M) \leq 3$ the invariant is related to classical invariants of the three manifold. So while one has a generalization of the Casson invariant, this generalization does not, at first sight, help to provide nontrivial invariants for three manifolds with $b_{1}(M) \neq 0$. One of the aims of this paper is to introduce observables which may correspond to non-trivial invariants for any $b_{1}(M)$.

In section 2 I give a quick review of the work of Rozansky and Witten, though the discussion presented is slightly different from the one in [16]. This is followed, in section 3, with a non-perturbative evaluation of the path integral for manifolds of the form $\Sigma \times S^{1}$. One obtains, in a straightforward way, the invariant $Z_{X}^{R W}\left[\Sigma \times S^{1}\right]$ in terms of invariants of $X$. One advantage of the approach adopted here is that one does not need to know that the Hilbert space of states is finite dimensional. The following section proceeds to slightly more complicated manifolds-mapping tori. This is followed by a discussion of the types of theories that one obtains on dimensional reduction. One finds B-type topological sigma models. Kapranov [8] and Kontsevich [10] have shown that the manifold $X$ need not be hyper-Kähler it is enough that it be holomorphic symplectic. Indeed the Kapranov-Kontsevich theory reduced yields a slight generalisation of the topological B-models.

There are a number of things that are missing. One has to do with the relationship of the Rozansky-Witten invariants to the known universal invariant the so called LMO invariant [13]. Rozansky and Witten establish that their theory provides a weight system, but this is just short of estab- 
lishing that their invariants arise from the LMO invariant. The missing part is given in [7], where a rather more general evaluation of the path integrals involved is also to be found. The relationship to the $S U(3)$ invariant [5] is also adressed there.

Another glaring gap is the relationship between this work and Donaldson theory on four manifolds of the form $M \times S^{1}$, the Seiberg-Witten invariant and the u-plane. Something which will be filled in elsewhere as it is one of the main motivations for this work.

\section{The Rozansky-Witten Model}

The starting point is that at low energies the path integral that corresponds to the Casson invariant becomes a topological supersymmetric sigma model. The supersymmetry is such that the target space of the sigma model should be hyper-Kähler. To write down such a topological field theory in three dimensions Rozansky and Witten twist a model that comes from the reduction of a supersymmetric sigma model in six dimensions. The way this works is reviewed presently. For the moment all we need are some facts about hyper-Kähler manifolds and their complexified tangent bundles.

A real manifold of dimension $2 m$ has holonomy group $S O(2 m)$ (or some subgroup thereof). If that manifold admits a complex structure then the holonomy group can be reduced to $\mathrm{U}(m) \subset \mathrm{S} O(2 m)$. Furthermore if the manifold admits a Ricci flat metric the holonomy group is $\mathrm{S} U(n)$. If $X$ is a hyper-Kähler manifold $\left(\operatorname{dim}_{\mathbb{R}} X=4 n\right)$ then there is a Riemannian metric such that the Levi-Cevita connection lies in an $\mathrm{S} p(n)$ subgroup of $\mathrm{S} O(4 n)$ (so that $\mathrm{S} p(n) \subseteq \mathrm{S} U(2 n) \subset \mathrm{U}(2 n) \subset \mathrm{S} O(4 n)$ ). The complexified tangent bundle decomposes as

$$
T X_{\mathbb{C}}=T X \otimes_{\mathbb{R}} \mathbb{C}=V \otimes S,
$$

where $V$ is a rank $2 n$ complex vector bundle with structure group $\mathrm{S} p(n)$ and $S$ is a trivial rank 2 complex vector bundle with structure group $\mathrm{S} p(1)$. The Levi-Cevita connection is a connection in $V$ and the trivial connection in $S$. Sp (1) labels are A, B, .. and there is an invariant antisymmetric tensor $\epsilon_{A B}$ with inverse $\epsilon^{A B}$,

$$
\epsilon^{A C} \epsilon_{C B}=\delta_{B}^{A}
$$

$\mathrm{S} p(n)$ labels are $\mathrm{I}, \mathrm{J}, \ldots$ and there is also an invariant antisymmetric tensor $\epsilon_{I J}$ with inverse $\epsilon^{I J}$,

$$
\epsilon^{I K} \epsilon_{K J}=\delta_{J}^{I}
$$


Local coordinates on $X$ will be denoted $\phi^{i}$ and the metric is $g_{i j}$. The fact that the tangent bundle decomposes as in (2.1) means that there exist covariantly constant tensors $\gamma_{i}^{A I}$ and $\gamma_{A I}^{i}$ that describe the maps from $V \otimes S$ to $T X_{\mathbb{C}}$ and vice versa. Using these tensors one may express the Riemann curvature tensor as

$$
R_{i j k l}=-\gamma_{i}^{A I} \gamma_{j}^{B J} \gamma_{k}^{C K} \gamma_{l}^{D L} \epsilon_{A B} \epsilon_{C D} \Omega_{I J K L}
$$

where $\Omega_{I J K L}$ is completely symmetric in the indices. A useful relationship is

$$
\epsilon_{I J} \gamma_{i}^{A I} \gamma_{j}^{B J}=\frac{1}{2} g_{i j} \epsilon^{A B}
$$

Fix on a complex structure so that $\phi^{I}$ are holomorphic coordinates on $X$ with respect to this complex structure. Then we may take

$$
\gamma_{A J}^{I}=\delta_{A 1} \delta_{J}^{I}, \quad \gamma_{A I}^{\bar{I}}=\delta_{A 2} g^{\bar{I} J} \epsilon_{J I}
$$

Notice that this means that we have essentially chosen $V_{\mathbb{C}}=T^{(1,0)} X . S$ is a trivial rank 2 bundle so that on picking a trivialisation one has $S=\mathbb{C} \oplus \mathbb{C}$. Now this means that $T_{\mathbb{C}} X=V \otimes S=V \otimes(\mathbb{C} \oplus \mathbb{C}) \equiv T^{(1,0)} X \oplus T^{(0,1)} X$.

With all the preliminaries out of the way we can write down the action. The action is

$$
S=c_{1} \int_{M} L_{1} \sqrt{h} d^{3} x+c_{2} \int_{M} L_{2}
$$

where

$$
\begin{aligned}
& L_{1}=\frac{1}{2} g_{i j} \partial_{\mu} \phi^{i} \partial^{\mu} \phi^{j}+\epsilon_{I J} \chi_{\mu}^{I} D^{\mu} \eta^{J} \\
& L_{2}=\frac{1}{2}\left(\epsilon_{I J} \chi^{I} D \chi^{J}+\frac{1}{3} \Omega_{I J K L} \chi^{I} \chi^{J} \chi^{K} \eta^{L}\right)
\end{aligned}
$$

The covariant derivative is

$$
D_{\mu J}^{I}=\partial_{\mu} \delta_{J}^{I}+\left(\partial_{\mu} \phi^{i}\right) \Gamma_{i J}^{I}
$$

Both of the Lagrangians $L_{1}$ and $L_{2}$ are invariant under two independent BRST supersymmetries. These supersymmetries are defined without the need of picking a prefered complex structure on $X$. We will not need this level of generality here, so pick a complex structure on $X$ so that the $\phi^{I}$ are 
local holomorphic coordinates with respect to this complex structure and let one of the BRST operators, $\bar{Q}$ act by

$$
\begin{aligned}
& \bar{Q} \phi^{I}=0, \quad \bar{Q} \phi^{\bar{I}}=g^{\bar{I} J} \epsilon_{J K} \eta^{K} \\
& \bar{Q} \eta^{I}=0, \quad \bar{Q} \chi^{I}=-d \phi^{I} .
\end{aligned}
$$

Since $L_{1}$ is BRST exact we may vary the coefficient $c_{1}$ with impunity. In particular one can take it, as the authors of [16] do, to equal $c_{2}$. There is an alternative choice which, from a different point of view, is more natural. If one starts with the four dimensional u-plane theory of Moore and Witten [15] on a four manifold of the form $M \times S^{1}$ where the circle has radius $R$ (tending to zero), then on reduction one would find $c_{1}=1 / R$ and $c_{2}=1$. As far as the partition function is concerned it is irrelevant as to which choice we make for the coefficients. However, the choices made affect the normalization of observables as will be seen below. For definiteness and to keep contact with [16] in most of what follows I will take, $c_{1}=c_{2}=c$.

\subsection{The Measure}

To completely specify the theory one needs to fix on some measure for the path integral. The theory under consideration does not have equality between Grassman odd and Grassman even fields, so it would seem that there is no canonical choice of measure for the path integral. However, there are a number of ways to see that the coupling constant dependence of the measure should be

$$
D\left(\phi^{i} \sqrt{c_{1}}\right) D\left(\eta^{I} \frac{c_{1}}{\sqrt{c_{2}}}\right) D\left(\chi^{I} \sqrt{c_{2}}\right)
$$

Firstly, this is the standard measure that one would choose given the kinetic terms in (2.7). Secondly, in the topological gauge theory that corresponds to the Casson invariant there is a precise pairing between Grassmann even and Grassmann odd fields which means that the measure in that theory is such that it does not have coupling constant dependence. Some of the fields that appear there are multiplier fields and these can be integrated out (algebraically eliminated). The net effect of integrating out the multiplier fields is to put the coupling constant into the measure of the remaining fields precisely as indicated in (2.12) (for the gauge theory). Also, thinking of $\mathrm{L}_{2}$ as a Chern-Simons type topological Lagrangian density and $\mathrm{L}_{1}$ as a gauge fixing Lagrangian one would be led to the measure (2.12), which guarantees that at one loop there is no coupling constant dependence. 
The choice of measure is important since it will be crucial for us to follow the coupling constant dependence as we take various limits. From now on set $c_{1}=c_{2}=c$.

Apart from factors of $c$ there are questions concerning the $\epsilon$ dependence of the measure. In [16] a detailed specification of this dependence is given. Also in the text some factors of $2 \pi$ make an appearance that is not commented on, for example in (3.21), these factors are obtained on scaling the relevant zero modes by $\sqrt{2 \pi}$ and are consistent with the normalization of the path integral given in [16].

\subsection{Reduction of 6-Dimensional Theories and Twisting}

The way that Rozansky and Witten derived the action (2.7) was to begin with a supersymmetric sigma model in six dimensions, to reduce to three and then to twist. The model in question has a map $\Phi: \mathbb{R}^{6} \rightarrow X$ and chiral fermions $\psi$ taking values in $\Phi^{*}(V)$. The $S O(6)(\operatorname{spin}(6))$ Lorentz symmetry for the bosons (fermions) in six dimensions becomes an $S O(3)_{E} \times S O(3)_{N}$ $\left(\left(S U(2)_{E} \times S U(2)_{N}\right) / \mathbb{Z}_{2}\right)$ symmetry on dimensional reduction ${ }^{3}$ to three dimensions, where $S O(3)_{E}$ is the Lorentz group on $\mathbb{R}^{3}$. The supersymmetry charges transform as two copies of $(\mathbf{2}, \mathbf{2})$ under $\left(S U(2)_{E} \times S U(2)_{N}\right)$. Such a supersymmetry cannot be placed on an arbitrary three manifold. To proceed one 'twists'. Twisting in this case means taking the diagonal subgroup $S U(2)_{E^{\prime}}$ of $\left(S U(2)_{E} \times S U(2)_{N}\right)$ to be the new Lorentz group. Under $S U(2)_{E^{\prime}}$ each copy of the supersymmetry charges transforms as $\mathbf{1} \oplus \mathbf{3}$. The tensorial properties of the fields can be determined. Note that under $S O(3)_{E} \times S O(3)_{N}$ the field $\Phi$ transforms as $(\mathbf{1}, \mathbf{1})$ under $S U(2)_{E^{\prime}}$ it is therefore a $\mathbf{1}$, so that it remains a zero form. The spinor $\psi$ transformed as $(\mathbf{2}, \mathbf{2})$ under $\left(S U(2)_{E} \times S U(2)_{N}\right) / \mathbb{Z}_{2}$ so that under the diagonal it transforms as $\mathbf{1} \oplus \mathbf{3}$, splitting into the zero form $\eta$ and the one form $\chi$.

The gauge theory that produces the Casson invariant is a twisted $N=4$ supersymmetric theory. This can also be obtained by reducing a theory from six dimensions down to three. The theory in question is $N=1$ super YangMills theory, which enjoys, in six dimensions, an extra 'accidental' $S U(2)_{R}$ symmetry. The field content in six dimensions is a connection $A_{M} d x^{M}$ and a chiral spinor $\psi$ (a section of $\mathcal{S}_{+} \otimes$ adP, where $\mathcal{S}_{+}$is the positive spin bundle). The supersymmetry charge, on reduction to three dimensions, transforms as a $(\mathbf{2}, \mathbf{2}, \mathbf{2})$ of $S U(2)_{E} \times S U(2)_{N} \times S U(2)_{R}$. As one can imagine there are many possible twisted theories, however the one of interest does not come by taking

\footnotetext{
${ }^{3}$ In the following discussion I will not be careful about wether the reduction includes a time like direction or not. Subtleties that arise from this have been adressed in [3].
} 
the diagonal of $S U(2)_{E} \times S U(2)_{N}$ as for the sigma model, rather, one sets $S U(2)_{E^{\prime}}$ to be the diagonal of $S U(2)_{E} \times S U(2)_{R}$. The supersymmetry charge remains a doublet of $S U(2)_{N}$, and transforms as a $\mathbf{1} \oplus \mathbf{3}$ of $S U(2)_{E^{\prime}}$. On reduction the connection transforms as $(\mathbf{3}, \mathbf{1}, \mathbf{1}) \oplus(\mathbf{1}, \mathbf{3}, \mathbf{1})$ while the spinor transforms as $(\mathbf{2}, \mathbf{2}, \mathbf{2})$ of $S U(2)_{E} \times S U(2)_{N} \times S U(2)_{R}$. On twisting, the connection transforms as $(\mathbf{3}, \mathbf{1}) \oplus(\mathbf{1}, \mathbf{3})$ and the spinor as a $(\mathbf{1}, \mathbf{2}) \oplus(\mathbf{3}, \mathbf{2})$ under $S U(2)_{E^{\prime}} \times S U(2)_{N}$.

It is curious that the two different path integral realizations of the Casson invariant arise on taking different twists of theories which start of life in six dimensions.

\subsection{The Perturbative Limit}

The fact that the theory does not depend on the constant $c$, means that we will be able to 'localise' the path integral onto the set of constant maps. Let,

$$
c=\frac{1}{R}
$$

with the aim of, shortly, taking the $R \rightarrow 0$ limit. Write the fields $\phi^{i}$ and $\eta^{I}$ in an orthogonal decomposition as 'fluctuating' parts with subscript $q$ (for quantum) plus harmonic components which have the subscript 0

$$
\begin{aligned}
\phi^{i} & =\phi_{0}^{i}+\phi_{q}^{i} \\
\eta^{I} & =\eta_{0}^{I}+\eta_{q}^{I},
\end{aligned}
$$

( $\chi^{I}$ can still be harmonic). In order to take the $R \rightarrow 0$ limit we scale the fluctuating fields

$$
\begin{aligned}
\phi^{i} & \rightarrow \phi_{0}^{i}+\sqrt{R} \phi_{q}^{i}, \\
\eta^{I} & \rightarrow \frac{1}{\sqrt{R}} \eta_{0}^{I}+\sqrt{R} \eta_{q}^{I}, \\
\chi^{I} & \rightarrow \sqrt{R} \chi^{I} .
\end{aligned}
$$

Notice that this scaling does not have unit Jacobian, rather the effect is to change the measure (2.12) to

$$
D\left(\phi^{i}\right) D\left(\eta^{I}\right) D\left(\chi^{I}\right) .
$$

On taking the limit the resulting Lagrangians are ${ }^{4}$

$$
\begin{aligned}
& { }^{4} \text { One also needs to shift } \\
& \qquad \eta^{I} \rightarrow \eta^{I}-\frac{1}{R} \phi^{i} \Gamma_{i J}^{I}\left(\phi_{0}\right) \eta_{0}^{J} .
\end{aligned}
$$




$$
\begin{aligned}
L_{1} \rightarrow & \frac{1}{2} g_{i j}\left(\phi_{0}\right) \partial_{\mu} \phi_{q}^{i} \partial^{\mu} \phi_{q}^{j}+\epsilon_{I J}\left(\phi_{0}\right) \chi_{\mu}^{I} \partial^{\mu} \eta_{q}^{J} \\
& -\frac{1}{2} \gamma_{i}^{A K} \gamma_{j}^{B L} \epsilon_{A B} \Omega_{I J K L}\left(\phi_{0}\right) \chi_{\mu}^{I} \eta_{0}^{J} \phi_{q}^{j} \partial^{\mu} \phi_{q}^{i} \\
L_{2} \rightarrow & \frac{1}{2}\left(\epsilon_{I J}\left(\phi_{0}\right) \chi^{I} d \chi^{J}+\frac{1}{3} \Omega_{I J K L}\left(\phi_{0}\right) \chi^{I} \chi^{J} \chi^{K} \eta_{0}^{L}\right)
\end{aligned}
$$

and these agree with the Lagrangians found in [16] which correspond to what they termed minimal Feynman diagrams ${ }^{5}$.

\subsection{Observables}

Rozansky and Witten introduced two types of observables. The first is made up of only the $\eta$ field and certain classes on $X$. There is an isomorphism between the spaces $\Omega^{(k, 0)}(X)$ and $\Omega^{(0, k)}(X)$ given by the tensor

$$
S \frac{I}{J}=\epsilon^{I J} g_{J \bar{J}}
$$

Let $\omega$ be a $k$-form which is $\partial$ closed as a $(k, 0)$-form and which is $\bar{\partial}$ closed as a $(0, k)$-form. From this and the similarity of the BRST operators to the Dolbeault operators one concludes that

$$
\mathcal{O}(\omega)=\omega_{I_{1}, \ldots, I_{k}}(\phi) \eta^{I_{1}} \ldots \eta^{I_{k}}
$$

is BRST closed and hence a good observable in the theory. From the scalings that we performed in the previous section we learn that these observables scale with an $R$ dependence, as

$$
\mathcal{O}(\omega) \mapsto\left(\frac{1}{\sqrt{R}}\right)^{k} \omega_{I_{1}, \ldots, I_{k}}\left(\phi_{0}\right) \eta_{0}^{I_{1}} \ldots \eta_{0}^{I_{k}}+\ldots
$$

where the ellipses indicate lower order terms. For example, when $n=1$ and $k=2$, the observable scales as

$$
\mathcal{O}(\omega)=\frac{1}{R} \omega_{I_{1}, I_{2}}\left(\phi_{0}\right) \eta_{0}^{I_{1}} \eta_{0}^{I_{2}}+\ldots
$$

These observables play a role in determining, from the path integral point of view, the behaviour of the Casson invariant under the operation of taking a connected sum [16]. The normalisation of the observable, as I have given

\footnotetext{
${ }^{5}$ With $c_{1}=1 / R$ and $c_{2}=1$ the required scalings to arrive at the minimal Feynman vertices are $\phi^{i} \rightarrow \phi_{0}^{i}+\sqrt{R} \phi_{q}^{i}$ and $\eta^{I} \rightarrow \eta_{0}^{I}+R \eta_{q}^{I}$.
} 
it here, therefore seems to be incorrect. Rather, one should perhaps define it from the outset to be

$$
\mathcal{O}(\omega)=(\sqrt{R})^{k} \omega_{I_{1}, \ldots, I_{k}}(\phi) \eta^{I_{1}} \ldots \eta^{I_{k}}
$$

though I have no convincing argument for this from the three dimensional viewpoint at present. On the other hand if we had begun with $c_{1}=1 / R$ and $c_{2}=1$ then after the scalings indicated in footnote 5 we would have found the more appealing result

$$
\mathcal{O}(\omega) \rightarrow \omega_{I_{1}, \ldots, I_{k}}\left(\phi_{0}\right) \eta_{0}^{I_{1}} \ldots \eta_{0}^{I_{k}}
$$

Let $\mathcal{K}$ be a knot and $\mathcal{R}$ a representation of $\operatorname{Sp}(n)$. The second set of observables is constructed from the gauge field,

$$
A_{I J}=d \phi^{i} \epsilon_{I K} \Gamma_{i J}^{K}+\Omega_{I J K L} \chi^{K} \eta^{L}
$$

Under a BRST transformation $A$ transforms as a gauge field ought to, so that Wilson loops,

$$
\mathcal{W}_{\mathcal{R}}(\mathcal{K})=\operatorname{Tr}_{\mathcal{R}} \mathrm{e}^{\oint_{\mathcal{K}} A}
$$

are BRST invariant and metric independent. Under the scalings of the last section (also for $c_{1}=1 / R$ and $c_{2}=1$ ) the gauge field goes over to

$$
A_{I J} \mapsto \Omega_{I J K L}\left(\phi_{0}\right) \chi^{K} \eta_{0}^{L}
$$

This connection will make an appearance again when we come to MappingTori.

\subsection{New Invariants and Beyond the $b_{1}(M)=3$ Barrier}

The problem that one faces in obtaining non-zero results past $b_{1}(M)=3$ is that there seems to be no natural way in which to handle the (excess) $\chi^{I}$ harmonic modes. For $\operatorname{dim}_{\mathbb{R}} X=4 n$ and $b_{1}(M)=b_{1}$ there are $2 n b_{1} \chi$ harmonic modes and $2 n \eta$ harmonic modes. The $\eta$ harmonic mode count tells us that the total number of insertions of vertices in any given diagram is $2 n$ so that that the most one can hope to do is to soak up $6 n \chi$ harmonic modes (since, as one can see from the vertices, there are at most three $\chi$ for each $\eta$ ). This puts the upper limit on $b_{1}$. Insertions of the observables do not help matters. In fact both the point observables $\mathcal{O}(\omega)$ and the knot observables $\mathcal{W}(\mathcal{K})$ work the wrong way in that they are $\eta$ dependent. 
Apart from the two types of observables that have already been discussed there is a third type which is available when $b_{1}(M) \neq 0$ and which can "soak up" the $\chi^{I}$ zero modes. However, the observables that we will construct are only invariant under one of the BRST operators. This should not be a problem in making sense of the observables. The usual arguments to prove metric independence of the expectation value of a product of observables only requires the existance of one BRST operator, $\bar{Q}$, with respect to which the observables in question are closed. If the observable is $\bar{Q}$ exact, then under normal conditions, its expectation value will vanish. If the $\bar{Q}$ invariant observable has non-zero expectation value then we may conclude that it is a non-trivial topological observable. The rôle of the other BRST operator is to pick out a representative of the $\bar{Q}$ class that the observable belongs to. The problem in explicitly constructing such a representative in the field theory is that it may have to be non-local. Of course, at the level of cohomology, any representative of a class is as good as any another so we do not need to impose the extra condition that observables are also $Q$ closed. Hence, I will take the attitude that since the observables below are in $\bar{Q}$ cohomology and are independent of the metric on $M_{3}$, that they constitute good topological observables.

The $\bar{Q}$ supersymmetry is such that given any closed 1-cycle $\gamma$

$$
\chi_{I}(\gamma)=\epsilon_{I J} \oint_{\gamma} \chi^{J},
$$

is invariant. The path integral is such that essentially the $\chi^{I}$ of interest will be harmonic on $M$ so that the $\gamma$ may as well be taken to live in $\mathrm{H}_{1}(M)$. For each $\gamma$ one may view $\chi_{J}(\gamma)$ as a section of $T^{(1,0)} X$. Let $\gamma_{i}, i=1, \ldots, b_{1}(M)$, be a basis of $H_{1}(M)$ and set

$$
\chi_{I}\left(\gamma_{i}\right)=\epsilon_{I J} \oint_{\gamma_{i}} \chi^{J} .
$$

One also has that

$$
\eta^{\bar{I}}=g^{\bar{I} J} \epsilon_{J K} \eta^{K}
$$

is $\bar{Q}$ invariant and the $\eta^{\bar{I}}$ are sections of $T^{(0,1)} X$.

Let $\lambda$ be a $(0, q)$ form on $X$ with values in $\wedge^{k_{1}} T^{(1,0)} X \otimes \wedge^{k_{2}} T^{(1,0)} X \otimes$ $\cdots \otimes \wedge^{k_{r}} T^{(1,0)} X$ where $b_{1}(M)=r$. In local coordinates such an object can be written as

$$
\begin{array}{r}
\lambda_{\bar{I}_{1} \bar{I}_{2} \ldots \bar{I}_{q}}^{\left(J_{1}^{1} \ldots J_{1}^{1}\right), \ldots,\left(J_{1}^{r} \ldots J_{k_{r}}^{r}\right)} d \bar{z}^{\bar{I}_{1}} \ldots d \bar{z}^{\bar{I}_{q}} \otimes \frac{\partial}{\partial z^{J_{1}^{1}}} \ldots \frac{\partial}{\partial z^{J_{k_{1}}^{1}}} \otimes \ldots \\
\cdots \otimes \frac{\partial}{\partial z^{J_{1}^{r}}} \ldots \frac{\partial}{\partial z^{J_{k_{r}}^{r}}} .
\end{array}
$$


In the above formula labels in a given set are understood to be antisymmetric amongst themselves. Now given such a form one can construct an observable

$$
\begin{aligned}
\mathcal{O}(\lambda)\left(q ; k_{1}, k_{2}, \ldots, k_{r}\right)= & \lambda_{\bar{I}_{1} \bar{I}_{2} \ldots \bar{I}_{q}}^{\left(J_{1}^{1} \ldots J_{k^{1}}^{1}\right), \ldots,\left(J_{1}^{r} \ldots J_{k_{r}}^{r}\right)} \eta^{\bar{I}_{1}} \ldots \eta^{\bar{I}_{q}} . \\
& \chi\left(\gamma_{1}\right)_{J_{1}^{1}} \ldots \chi\left(\gamma_{1}\right)_{J_{k_{1}}^{1}} \\
& \ldots \chi\left(\gamma_{r}\right)_{J_{1}^{r}} \ldots \chi\left(\gamma_{r}\right)_{J_{k_{r}}^{r}}
\end{aligned}
$$

One may well need to average over cycles in computing expectation values of such observables to ensure that the outcome does not depend on the choices of basis that we have made.

Perhaps the prototypical example of such an observable is that made out of the holomorphic symplectic two form itself. One defines

$$
\epsilon\left(\gamma_{1}, \gamma_{2}\right)=\epsilon_{I J} \oint_{\gamma_{1}} \chi^{I} \oint_{\gamma_{2}} \chi^{J}
$$

which is BRST invariant by virtue of the fact that $\epsilon_{I J}$ is holomorphic. Products of such operators are also BRST invariant and hence good observables. Each such operator soaks up 2 of the $\chi$ zero modes so that if there are $m$ such operators then one finds the 'selection rule'

$$
6 n+2 m=2 b_{1} n
$$

which acts as an upper bound on $b_{1}$ so that the expectation value of the observable does not necessarily vanish.

Let $\lambda=\epsilon^{n}$ and note that $\lambda \in \Omega^{(0,0)}\left(M, \wedge^{2 n} T^{(1,0)} X\right)$ so that it is of the type that we have introduced above. One may define observables

$$
\mathcal{O}(\lambda)\left(\gamma_{i}\right)=\left(\epsilon_{I J} \oint_{\gamma_{i}} \chi^{I} \oint_{\gamma_{i}} \chi^{J}\right)^{n}
$$

For example let $b_{1}=4$, then we need $m=n$. In fact a nice example is $n=m=2$.

These observables may give rise to more effective invariants of three manifolds with $b_{1}>0$ than the Rozansky-Witten invariants. As discussed in the introduction $Z_{X}^{R W}[M]$ is, for all hyper-Kähler manifolds $X$, essentially a classical invariant of $M$ if $b_{1}(M)>0$. How one deals with the Rozansky-Witten invarant in perturbation theory depends on $b_{1}(M)$ through the number of $\chi^{I}$ harmonic modes. Insertions of operators that only involve the $\chi^{I}$ harmonic modes means that the perturbation series is effectively the same as that for a three manifold with smaller first betti-number. Inserting enough operators 
into the path integral will mean that the invariant will be proportional to the same type of integrals of products of Greens functions that are typical of the Casson invariant for a three manifold with $b_{1}=0$.

The choice of normalization of operators is important here as well. With the normalization above and the scalings of the previous section these observables will vanish as $R \rightarrow 0$. For the choice $c_{1}=1 / R$ and $c_{2}=1$ the scalings given in footnote 5 tell us to replace the fields with their harmonic modes and in the $R \rightarrow 0$ limit the operators survive.

\subsection{Ray-Singer Torsion and Duality}

There is a small puzzle that presents itself. The twisted $N=4$ theory will calculate the Casson invariant. One can twist the physical low energy theory and once more one should be calculating the Casson invariant. But now we seem to have gotten more than our moneys worth as not only does one find the Casson invariant but at one loop the theory also yields up the Ray-Singer torsion. How can this be?

The answer to this is that there is a difference between the topological theory that one finds by twisting the low energy effective physical theory and the Rozansky-Witten model. They differ by dualising the gauge field. The path integral for a photon and that for a (compact scalar) on $\mathbb{R}^{3}$ agree, however, on an arbitrary three manifold the ratio of path integrals is precisely the Ray-Singer torsion. The easiest way to see this is to simply take the ratio. The path integral for the gauge field gives

$$
\operatorname{det} \Delta_{1}^{-1 / 2} \cdot \operatorname{det} \Delta_{0}
$$

while that for the scalar is

$$
\operatorname{det} \Delta_{0}^{-1 / 2}
$$

where the subscript denotes the form degree that the Laplacian is acting on. The required ratio is

$$
\operatorname{det} \Delta_{1}^{-1 / 2} \cdot \operatorname{det} \Delta_{0}^{3 / 2}
$$

which is (the inverse of) the Ray-Singer Torsion. So on dualising the topological gauge theory to obtain the topological sigma model one is feeding in the one loop Ray-Singer Torsion. 


\section{Calculations on $\Sigma_{g} \times S^{1}$}

When the three manifold is a product of a Riemann surface $\Sigma_{g}$, of genus $g$, and a circle one can explicitly evaluate the Rozansky-Witten path integral. The actions on such a manifold are,

$$
\begin{aligned}
S_{1}= & \int_{\Sigma_{g} \times S^{1}} \\
& \sqrt{h_{2}} \frac{1}{2} g_{i j}\left(\partial_{\mu} \phi^{i} \partial^{\mu} \phi^{j}+\partial_{t} \phi^{i} \partial_{t} \phi^{j}\right) \\
& +\epsilon_{I J}\left(\chi_{\mu}^{I} D^{\mu} \eta^{J}+\bar{\eta}^{I} D_{t} \eta^{J}\right) \\
S_{2}= & \int_{\Sigma_{g} \times S^{1}}\left(\epsilon_{I J}\left(\bar{\eta}^{I} D \chi^{J}-\frac{1}{2} \chi^{I} D_{t} \chi^{J}\right)+\Omega_{I J K L} \chi^{I} \chi^{J} \bar{\eta}^{K} \eta^{L}\right)
\end{aligned}
$$

where $x^{\mu}$ are local co-ordinates on $\Sigma_{g}$, I have taken a product metric

$$
h_{3}=h_{2} \oplus d t^{2} \text {, }
$$

and have set $\chi_{t}^{I}=\bar{\eta}^{I}$.

In order to proceed we will need to scale the metric of the Riemann surface down so as to obtain an effective super quantum mechanics theory. Keep the overall coefficient (2.13) as is but scale the metric

$$
d s^{2}=R h_{\mu \nu} d x^{\mu} \otimes d x^{\nu} \oplus d t^{2},
$$

so that this limit is slightly different to the one we adopted for the perturbative calculations. Of course $S_{2}$ does not feel these choices, but $S_{1}$ certainly does. In order to be able to take the $R \rightarrow 0$ limit one needs to scale the fields. Let a 0 subscript denote the part of the fields which are harmonic on the $\Sigma_{g}$ and fields with a $\perp$ subscript are orthogonal to these, with respect to the metric on $\Sigma_{g}$. The scalings that are to be made are the following

$$
\begin{aligned}
\phi^{i} & \rightarrow \phi_{0}^{i}+\sqrt{R} \phi_{\perp}^{i} \\
\chi^{I} & \rightarrow \sqrt{R}\left(\chi_{0}^{I}+\chi_{\perp}^{I}\right) \\
\eta^{I} & \rightarrow \eta_{0}^{I}+\sqrt{R} \eta_{\perp}^{I} \\
\bar{\eta}^{I} & \rightarrow \bar{\eta}_{0}^{I}+\sqrt{R} \bar{\eta}_{\perp}^{I} .
\end{aligned}
$$

After these scalings the limit $R \rightarrow 0$ can be taken. Once more the choice of scaling is so that the measure is now

$$
D\left(\phi^{i}\right) D\left(\eta^{I}\right) D\left(\bar{\eta}^{I}\right) D\left(\chi^{I}\right) .
$$

Let us look at each term seperately. As far as $c S_{1}$ is concerned the bosonic field kinetic energy terms are well behaved giving

$$
\int_{\Sigma_{g} \times S^{1}} \sqrt{h_{2}} \frac{1}{2} g_{i j}\left(\phi_{0}\right)\left(\partial_{\mu} \phi_{\perp}^{i} \partial^{\mu} \phi_{\perp}^{j}+\partial_{t} \phi_{0}^{i} \partial_{t} \phi_{0}^{j}\right) .
$$


There are also the fermionic terms

$$
\begin{aligned}
& \frac{1}{R} \int_{\Sigma_{g} \times S^{1}} \sqrt{h_{2}} \epsilon_{I J}\left(\phi_{0}\right) \chi_{\mu}^{I} D^{\mu} \eta^{J} \\
& \quad \rightarrow \int_{\Sigma_{g} \times S^{1}} \sqrt{h_{2}} \epsilon_{I J}\left(\phi_{0}\right) \chi_{\mu}^{I} \partial^{\mu}\left(\eta_{\perp}^{J}+\phi_{\perp}^{i} \Gamma_{i K}^{J}\left(\phi_{0}\right) \eta_{0}^{K}\right)
\end{aligned}
$$

and

$$
\frac{1}{R} \int_{\Sigma_{g} \times S^{1}} \sqrt{h_{2}} \epsilon_{I J} \bar{\eta}^{I} D_{t} \eta^{J} \rightarrow \int_{\Sigma_{g} \times S^{1}} \sqrt{h_{2}} \epsilon_{I J}\left(\phi_{0}\right) \bar{\eta}_{0}^{I} D_{t}\left(\phi_{0}\right) \eta_{0}^{J} .
$$

Now turn to $c S_{2}$. We have,

$$
\begin{aligned}
\frac{1}{R} \int_{\Sigma_{g} \times S^{1}} \frac{1}{2} \epsilon_{I J} \chi^{I} D_{t} \chi^{J} & \rightarrow \int_{\Sigma_{g} \times S^{1}} \frac{1}{2} \epsilon_{I J}\left(\phi_{0}\right) \chi^{I} D_{t}\left(\phi_{0}\right) \chi^{J} \\
\frac{1}{R} \int_{\Sigma_{g} \times S^{1}} \Omega_{I J K L} \chi^{I} \chi^{J} \bar{\eta}^{K} \eta^{L} & \rightarrow \int_{\Sigma_{g} \times S^{1}} \Omega_{I J K L} \chi^{I} \chi^{J} \bar{\eta}_{0}^{K} \eta_{0}^{L}
\end{aligned}
$$

and

$$
\begin{aligned}
& \frac{1}{R} \int_{\Sigma_{g} \times S^{1}} \epsilon_{I J} \bar{\eta}^{I} D \chi^{J} \\
& \quad \rightarrow \int_{\Sigma_{g} \times S^{1}}\left(\epsilon_{I J}\left(\phi_{0}\right) \bar{\eta}_{\perp}^{I}+\bar{\eta}_{0}^{I} \epsilon_{L J}\left(\phi_{0}\right) \phi_{\perp}^{K} \Gamma_{K I}^{L}\left(\phi_{0}\right)\right) d \chi^{J} .
\end{aligned}
$$

So the combined actions (3.7) to (3.12) are what we end up with. However, one can simplify matters greatly by noticing that the path integrals over $\bar{\eta}_{\perp}^{I}$ and $\eta_{\perp}^{I}$ imply that $\chi^{I}$ is harmonic on $\Sigma_{g}$. We can feed this back in to the actions above to arrive at rather more simplified expressions.

The action that one obtains on taking the limit is the sum of the following two

$$
\begin{aligned}
S_{0}=\oint d & \left(\frac{1}{2} g_{i j}\left(\phi_{0}\right) \partial_{t} \phi_{0}^{i} \partial_{t} \phi_{0}^{j}+\epsilon_{I J}\left(\phi_{0}\right) \bar{\eta}_{0}^{I} D_{t} \eta_{0}^{J}\right. \\
& \left.-\frac{1}{2} \epsilon_{I J}\left(\phi_{0}\right) \chi_{0}^{I} D_{t}\left(\phi_{0}\right) \chi_{0}^{J}+\Omega_{I J K L} \chi_{0}^{I} \chi_{0}^{J} \bar{\eta}_{0}^{K} \eta_{0}^{L}\right)
\end{aligned}
$$

and

$$
\begin{gathered}
S_{\perp}=\int_{\Sigma_{g} \times S^{1}}\left[\sqrt{h_{2}}\left(\frac{1}{2} g_{i j}\left(\phi_{0}\right) \partial_{\mu} \phi_{\perp}^{i} \partial^{\mu} \phi_{\perp}^{j}+\epsilon_{I J}\left(\phi_{0}\right) \chi_{\perp \mu}^{I} D^{\mu}\left(\phi_{0}\right) \eta_{\perp}^{J}\right)\right. \\
\left.+\epsilon_{I J}\left(\phi_{0}\right) \bar{\eta}_{\perp}^{I} D\left(\phi_{0}\right) \chi_{\perp}^{J}\right]
\end{gathered}
$$


Notice that $S_{0}$ is a standard, topological, supersymmetric quantum mechanics action.

After all these manouvers one obtains the partition function,

$$
Z_{X}^{R W}\left[\Sigma_{g} \times S^{1}\right]=\int D \phi_{0}^{i} D \eta_{0}^{I} D \bar{\eta}_{0}^{I} D \chi_{0}^{I} \mathrm{e}^{-\lambda S_{0}} Z_{\perp}\left[\Sigma_{g} \times S^{1}\right]
$$

where

$$
Z_{\perp}=\int D \phi_{\perp}^{i} D \chi_{\perp}^{I} D \eta_{\perp}^{I} D \bar{\eta}_{\perp}^{I} \mathrm{e}^{-S_{\perp}}
$$

and $\lambda$ is the volume of the $\Sigma_{g}$ with respect to the metric $h_{2}$. The partition function (3.16) is unity. As explained in [16] this partition function calculates the Ray-Singer torsion. Since we are integrating over modes which are not harmonic on $\Sigma_{g}$ the cohomology that is being seen on $\Sigma_{g} \times S^{1}$ is essentially acyclic. In this case the Ray-Singer torsion is honestly 'trivial' and the partition function is unity.

We are left with the following partition function

$$
Z_{X}^{R W}\left[\Sigma_{g} \times S^{1}\right]=\int D \phi_{0}^{i} D \eta_{0}^{I} D \bar{\eta}_{0}^{I} D \chi_{0}^{I} \mathrm{e}^{-\lambda S_{0}}
$$

\subsection{The Path Integral on $S^{2} \times S^{1}$}

On the two sphere there are no $\chi^{I}$ harmonic modes so that the path integral becomes

$$
Z_{X}^{R W}\left[S^{2} \times S^{1}\right]=\int D \phi_{0}^{i} D \eta_{0}^{I} D \bar{\eta}_{0}^{I} \mathrm{e}^{-\lambda S_{0}^{\prime}}
$$

where

$$
S_{0}^{\prime}=\oint d t\left(\frac{1}{2} g_{i j}\left(\phi_{0}\right) \partial_{t} \phi_{0}^{i} \partial_{t} \phi_{0}^{j}+\epsilon_{I J}\left(\phi_{0}\right) \bar{\eta}_{0}^{I} D_{t} \eta_{0}^{J}\right)
$$

Happily enough, we do not have to evaluate this path integral ${ }^{6}$. When $X$ is compact and hyper-Kähler this is the supersymmetric quantum mechanics

\footnotetext{
${ }^{6}$ However, it is not difficult to do so. Following standard calculations as in [1] one obtains the second equality in (3.20) as it is given. One thing I made use of though, is the isomorphism between the holomorphic and anti-holomorphic tangent bundles. This is achieved by the covariantly constant, non-degenerate, tensor $S_{J}^{\bar{I}}=g^{K \bar{I}} \epsilon_{K I}$. This means that one may exchange $\epsilon_{I J} \bar{\eta}^{J}$ for $g_{I \bar{J}} \bar{\eta}^{\bar{J}}$ in the supersymmetric quantum mechanics theory.
} 
path integral that evaluates (minus ${ }^{7}$ ) the index of the Dolbeault operator $[1,22]$. When $X$ is hyper-Kähler but not compact, one finds the same combination of Riemann tensor terms as in the compact case (though this need no longer be the 'index' of the Dolbeault operator), hence ${ }^{8}$

$$
\begin{aligned}
Z_{X}^{R W}\left[S^{2} \times S^{1}\right] & =-\int_{X} \operatorname{Todd}\left(T X_{\mathbb{C}}\right) \\
& =-\int_{X} \prod_{i} \frac{\left(x_{i} / 2\right)}{\sinh \left(x_{i} / 2\right)},
\end{aligned}
$$

where the $x_{i}$ are the eigenvalues of

$$
R_{I \bar{J}}=\frac{i}{2 \pi} R_{I \bar{J} K \bar{L}} d z^{K} d \bar{z}^{\bar{L}} .
$$

This calculation corroborates that in [16] which uses a Hilbert space approach to determine the partition function. The advantage that we have here is that we do not deal with the cohomology groups directly and so do not have to identify $Z_{X}\left[S^{2} \times S^{1}\right]$ with $\sum_{k=0}^{2 n}(-1)^{k+1} \operatorname{dim} H^{(0, k)}$, though this identification is correct for $X$ compact.

\subsection{The Path Integral on $T^{2} \times S^{1}$}

The sigma model in this case is rather easy to get a handle on. Essentially one can forget all the non-harmonic modes so that the final 'path integral' is an integral over $X$ plus integration over the fermions. The formula that one obtains is precisely that that one would obtain from supersymmetric quantum mechanics for the index of the de-Rham operator, that is the integral of the Euler class.

In detail one sees that on $T^{2}$ there are two $\chi^{I}$ harmonic modes, $\chi_{1}^{I}$ and $\chi_{2}^{I}$. One can combine fields in the following way

$$
\begin{aligned}
& \psi=\left(\chi_{1}^{I}, g^{\bar{I} K} \epsilon_{K J} \bar{\eta}_{0}^{J}\right) \\
& \bar{\psi}=\left(\eta_{0}^{I}, g^{\bar{I} K} \epsilon_{K J} \chi_{2}^{J}\right),
\end{aligned}
$$

\footnotetext{
${ }^{7}$ The sign is not easy to determine apriori. However, with sign for the calculations on $T^{3}$ given, the sign here follows from the calculations performed on Mapping-Tori.

${ }^{8}$ The definition of the Todd class is

$$
\prod_{i} \mathrm{e}^{\left(x_{i} / 2\right)} \frac{\left(x_{i} / 2\right)}{\sinh \left(x_{i} / 2\right)}=\mathrm{e}^{\sum_{i}\left(x_{i} / 2\right)} \prod_{i} \frac{\left(x_{i} / 2\right)}{\sinh \left(x_{i} / 2\right)}=\mathrm{e}^{c_{1}(X) / 2} \prod_{i} \frac{\left(x_{i} / 2\right)}{\sinh \left(x_{i} / 2\right)}
$$

but for the manifolds in question one has $c_{1}(X)=0$. This means that, $\hat{\mathrm{A}}\left(T X_{\mathbb{C}}\right)=$ $\operatorname{Todd}\left(T X_{\mathbb{C}}\right)$ a familiar fact for Calabi-Yau manifolds.
} 
and the action in these variables is precisely that of the topological field theory that calculates the Euler characteristic of $X$ ! The path integral presents us with the Euler characteristic in Gauss-Bonnet form. Hence, regardless of whether $X$ is compact or not, the final formula for the path integral is as an integral over $X$ of the Euler class. Again this agrees with the calculations in [16] for compact manifolds, namely that

$$
\begin{aligned}
Z_{X}^{R W}\left[T^{3}\right] & =\operatorname{Str} \oplus_{i, j=0}^{2 n} H^{(i, j)}(X) \\
& =\sum_{i, j=0}^{2 n}(-1)^{i+j} b^{(i, j)}(X) \\
& =\chi(X)
\end{aligned}
$$

Now the Riemann curvature two-form $R_{b}^{a}$ is self dual for both $K 3$ and $X_{A H}$, indeed for all hyper-Kähler four manifolds, so that the ratio of integrals that formally represent the Euler characteristic and the $\hat{\mathrm{A}}$ genus do not depend on the hyper-Kähler manifold in question. This is one of the main properties exploited in [16] so as to use results from the compact manifolds to arrive at equivalent statements for the non-compact ones. Of course when the real dimension of $X$ is greater than 4 , the situation becomes somewhat more involved.

\subsection{The $\chi$ Path Integral}

The number of components of $\chi_{0}$ depends on the genus $g$ of $\Sigma_{g}$. For the sphere there are no such modes and the path integral calculated the integral of the Todd class of $X$. This, for a compact manifold, coincides with the index of the Dolbeault operator $\bar{\partial}$ on $X$. So the $\eta, \bar{\eta}$ path integral can be said to correspond to the $\bar{\partial}$ operator. On the Torus one found instead, owing to the presence of two $\chi_{0}$ zero-modes, that the path integral yields the integral over $X$ of the Euler class. This for compact $X$ corresponds to the index of the de-Rham operator. Alternatively this may also be viewed as the index of the Dolbeault operator with values in $\mathrm{T}^{(0,1)} X$. From this second, character valued, viewpoint the $\eta, \bar{\eta}$ system still corresponds to the Dolbeault operator, and the $\chi$ fields are there to take into account the fact that the forms take values in $\mathrm{T}^{(0,1)} X$.

For genus $g$ the $\chi$ system corresponds to forms taking values in $\left(\wedge \mathrm{T}^{(0,1)} X\right)^{\otimes g}$. Consequently, the path integral for compact $X$ calculates the 
super dimension of

$$
\mathcal{H}_{\Sigma_{g}}=\sum_{i}^{\operatorname{dim}_{\mathbb{C}} X} H^{(i, 0)}\left(X,\left(\wedge \mathrm{T}^{(0,1)} X\right)^{\otimes g}\right)
$$

where the $H^{(i, 0)}\left(X,\left(\wedge \mathrm{T}^{(0,1)} X\right)^{\otimes g}\right)$ are the Dolbeault cohomology groups of $X$ with values in $\left(\wedge \mathrm{T}^{(0,1)} X\right)^{\otimes g}$. The path integral gives the index of the operator in terms of powers of the Riemann curvature tensor. When $X$ is non-compact precisely the same combinations of the Riemann curvature tensor appear. The only thing lacking is the interpretation of this object as the index of the Dolbeault operator.

In either case ( $X$ compact or not) for $g>1$ the path integral vanishes. We saw this in the perturbative expansion and it is unfortunate, but still true in the current setting since we can still only pull down $R^{2 n}$ (because of $\eta$ zero modes). It is unfortunate for while one sees quite directly that the relevant Hilbert space (for $X$ compact) is (3.24) the usefulness, at the moment, of this remains academic as the path integral vanishes. The fact that the path integral vanishes is that the integral is over $X$ but the 'form degree' is greater than $4 n$ (measured now by the number of $\eta, \bar{\eta}$ modes and half of the $\chi$ 's).

The details of the supersymmetric path integral approach to the index theorem with values in a bundle (the $\chi$ integral) can be found, for example, in $[1]$.

\section{Calculations on Mapping Tori}

In this section I will generalize slightly the three manifolds that can be dealt with by shrinking a Riemann surface away. Here we will be interested in Mapping Tori over a circle. A Mapping Torus is a three manifold that is constructed from $\Sigma_{g} \times[0,1]$ on gluing the two $\Sigma_{g}$ boundaries together after acting on one of them by a diffeomorphism $f$.

The path integral on such a manifold is the same as a path integral on $\Sigma_{g} \times \mathbb{R}$ but with all fields $\Phi$ satisfying

$$
\Phi(x, t+1)=f^{*} \Phi(x, t) .
$$

We still have the freedom, in the Rozansky-Witten model, to take the zero volume limit of $\Sigma_{g}$ in the path integral on $\Sigma_{g} \times[0,1]$. This will once more 'squeeze' away all the states on $\Sigma_{g}$ except for the harmonic modes. Hence, 
the path integral on $\Sigma_{g} \times[0,1]$ with the boundary conditions (4.1) will devolve to a path integral on the circle with the insertion of an operator that implements the diffeomorphism on the harmonic modes. Denote this operator by $U$ and denote the Mapping Torus by $\Sigma_{U}$.

What is $U$ ? We only need to ask how $f$ acts on the $\chi_{0}^{I}$ fields since it acts trivially on $\eta_{0}^{I}$ and $\bar{\eta}_{0}^{I}$. Let $a_{\alpha}$, and $b_{\alpha}$, for $\alpha=1, \ldots, g$, be a "canonical" basis for $H_{1}\left(\Sigma_{g}, \mathbb{Z}\right) \cong \mathbb{Z}^{2 g}$, such that the intersection pairing of the cycles satisfies

$$
\left(a_{\alpha}, a_{\beta}\right)=\left(b_{\alpha}, b_{\beta}\right)=0
$$

and

$$
\left(a_{\alpha}, b_{\beta}\right)=-\left(b_{\alpha}, a_{\beta}\right)=\delta_{\alpha \beta}
$$

Let $\omega_{a}^{\alpha}$ and $\omega_{b}^{\alpha}$ be a basic set of real harmonic 1-forms dual to the homology basis

$$
\int_{a_{\beta}} \omega_{a}^{\alpha}=\int_{b_{\beta}} \omega_{b}^{\alpha}=\delta^{\alpha \beta}, \quad \int_{a_{\beta}} \omega_{b}^{\alpha}=\int_{b_{\beta}} \omega_{a}^{\alpha}=0
$$

and

$$
\int_{\Sigma} \omega_{a}^{\alpha} \wedge \omega_{b}^{\beta}=\sum_{\gamma=1}^{g}\left(\int_{a_{\gamma}} \omega_{a}^{\alpha} \int_{b_{\gamma}} \omega_{b}^{\beta}-\int_{a_{\gamma}} \omega_{b}^{\beta} \int_{b_{\gamma}} \omega_{a}^{\alpha}\right)=\delta^{\alpha \beta}
$$

The mapping class group acts on the homology basis by elements of $\mathrm{S} p(2 g, \mathbb{Z})$. Let

$$
E=\left(\begin{array}{cc}
0 & \mathbb{I} \\
-\mathbb{I} & 0
\end{array}\right)
$$

a $2 g \times 2 g$ matrix. Then $U \in \mathrm{S} p(2 g, \mathbb{Z})$ means that

$$
U^{\mathrm{T}} . E . U=E,
$$

and one has

$$
U=\left(\begin{array}{ll}
A & B \\
C & D
\end{array}\right)
$$

where

$$
\begin{aligned}
A^{\mathrm{T}} C-C^{\mathrm{T}} A & =0 \\
B^{\mathrm{T}} D-D^{\mathrm{T}} B & =0 \\
A^{\mathrm{T}} D-C^{\mathrm{T}} B & =\mathbb{I} .
\end{aligned}
$$


Now expand $\chi_{0}^{I}$ in the basis,

$$
\chi_{0}^{I}=\chi_{\alpha}^{I a}(t) \omega_{a}^{\alpha}+\chi_{\alpha}^{I b}(t) \omega_{b}^{\alpha},
$$

where the $\chi_{\alpha}^{I}$ are fields on the $S^{1}$. By virtue of (4.4) the action of the mapping class group, thought of as acting on the $\chi^{I}$ is exactly the same as on the homology basis. For $U \in \mathrm{S} p(2 g, \mathbb{Z})$ the action on $\chi^{I}$ is

$$
\left(\begin{array}{c}
\chi_{a}^{I} \\
\chi_{b}^{I}
\end{array}\right) \rightarrow\left(\begin{array}{c}
A \chi_{a}^{I}+B \chi_{b}^{I} \\
C \chi_{a}^{I}+D \chi_{b}^{I}
\end{array}\right)
$$

\subsection{The Path Integral}

The path integral to be performed,

$$
Z_{X}^{R W}\left[\Sigma_{U}\right]=\int D \phi^{i} D \eta^{I} D \bar{\eta}^{I} \int_{U} D \chi^{I} \mathrm{e}^{-\lambda S_{0}}
$$

has the same form as (3.17) except that the $\chi^{I}$ are now not periodic on the circle but rather satisfy twisted boundary conditions. The zero subscript on the fields has been dropped as it is clear that this path integral is on the circle and all tensors on $X$ are understood to depend on $\phi_{0}$. The path integral measure is

$$
\left(D \phi^{i} \sqrt{\lambda}\right)\left(D \eta^{I} \sqrt{\lambda}\right)\left(D \bar{\eta}^{I} \sqrt{\lambda}\right)\left(D \chi^{I} \sqrt{\lambda}\right) .
$$

We expand the fields in Fourier modes on the circle and perform that following scalings; for $\phi^{i}, \eta^{I}$ and $\bar{\eta}^{I}$ all their modes except the constant mode are scaled (divided) by $\sqrt{\lambda}$, all the modes of $\chi^{I}$ are scaled by $\sqrt{\lambda}$. The measure is now $\lambda$ independent and the path integral factorises as

$$
Z_{X}^{R W}\left[\Sigma_{U}\right]=\int D \phi^{i} D \eta^{I} D \bar{\eta}^{I} \mathrm{e}^{-S^{\prime}} \int_{U} D \chi^{I} \mathrm{e}^{-S} \chi
$$

where

$$
\begin{aligned}
S_{\chi} & =\oint\left(\frac{1}{2} \epsilon_{I J} \chi^{I} \partial_{t} \chi^{J}+\Omega_{I J K L} \chi^{I} \chi^{J} \bar{\eta}_{0}^{K} \eta_{0}^{L}\right) \\
& =\oint\left(\frac{1}{2} \epsilon_{I J}\left(\chi^{\mathrm{T}}\right)^{I} \cdot E \cdot \partial_{t} \chi^{J}+\Omega_{I J K L}\left(\chi^{\mathrm{T}}\right)^{I} \cdot E \cdot \chi^{J} \bar{\eta}_{0}^{K} \eta_{0}^{L}\right)
\end{aligned}
$$

and the $\eta_{0}^{I}, \bar{\eta}_{0}^{K}$ are the constant modes of the fields.

It is not completely straightforward to evaluate the $\chi$ path integral. The procedure that I will follow is to change variables from the $\chi^{I}$ fields to a 
periodic set of fields $\psi^{I}$. The price to be paid is that a new connection appears in the action for the $\psi^{I}$ fields. Let

$$
\chi^{I}(t)=\mathrm{e}^{i t v} \cdot \psi^{I}(t)
$$

where

$$
\mathrm{e}^{i v}=U
$$

As one varies $t$, the matrix $\mathrm{e}^{i t v}$ runs along a path in $S p(2 g, \mathbb{R})$ from the identity to $U \in S p(2 g, \mathbb{Z})$. The field $\psi^{I}$ is periodic in $t$, so that

$$
\begin{aligned}
\chi^{I}(t+n) & =\mathrm{e}^{i(t+n) v} \cdot \psi^{I}(t) \\
& =U \cdot \chi^{I}(t+n-1),
\end{aligned}
$$

as required on the Mapping Torus. The action (4.15), in terms of the new variables is,

$$
S_{\psi}=\oint\left(\psi^{\mathrm{T}}\right)^{I} \cdot E \cdot\left(\frac{1}{2} \epsilon_{I J}\left(\partial_{t}+i v\right)+\Omega_{I J K L} \bar{\eta}_{0}^{K} \eta_{0}^{L}\right) \psi^{J},
$$

so that the path integral goes over to

$$
\int_{U} D \chi^{I} \mathrm{e}^{-S_{\chi}}=\int D \psi^{I} \mathrm{e}^{-S_{\psi}}
$$

Formally this path integral evaluates the square root of the determinant (Pfaffian) of the operator

$$
\left(\partial_{t}+i v\right) \delta_{J}^{I}+R_{J K L}^{I} \bar{\eta}_{0}^{K} \eta_{0}^{L},
$$

which is the (pull-back of the) covariant derivative on sections of an $\mathrm{S} p(2 g, \mathbb{R}) \otimes$ $\mathrm{S} p(n)$ bundle. One may write the operator then as

$$
\partial_{t}+A
$$

where

$$
A=v \otimes \mathbb{I} \oplus \mathbb{I} \otimes R .
$$

In (4.23) $R$ is the matrix form,

$$
R_{I \bar{J}}(\eta, \bar{\eta})=\frac{i}{2 \pi} R_{I \bar{J} K \bar{L}} \eta^{K} \bar{\eta}^{\bar{L}} .
$$

Notice that the $\operatorname{Sp}(n)$ part of the gauge field is precisely the component of $(2.29)$ in the time direction. 
To proceed I will evaluate the determinant and then take its square root. One way to do this is to work on the interval and to specify boundary data. This approach is explained in a related context ${ }^{9}$ in section (3.1) of [4].

By following the derivation in [4] one obtains

$$
\operatorname{Det}\left(U \otimes \mathbb{I}-\mathbb{I} \otimes \mathrm{e}^{R(\eta, \bar{\eta})}\right)^{1 / 2}
$$

which agrees with (the equivalent of) (3.24) of [4] when $U=I$. The path integral over the non-constant modes of $\phi^{i}, \eta^{I}$ and $\bar{\eta}^{I}$ give back the integrand of the $S^{2} \times S^{1}$ path integral (3.20) but with the $x_{i}$ now the eigenvalues of (4.24). The integral over the constant modes of $\eta^{I}$ and $\bar{\eta}^{\bar{I}}$ turn the $x_{i}$ into the eigenvalues of (3.21). So, putting all the pieces together we obtain

$$
Z_{X}^{R W}\left[\Sigma_{U}\right]=-\int_{X} \operatorname{Todd}\left(T X_{\mathbb{C}}\right) \operatorname{Det}\left(U \otimes I-I \otimes \mathrm{e}^{R}\right)^{1 / 2}
$$

One can now write this as

$$
Z_{X}^{R W}\left[\Sigma_{U}\right]=c_{X} I\left[\Sigma_{U}\right]
$$

To obtain the coefficient, $I\left[\Sigma_{U}\right]$, of the top form one essentially differentiates the integrand, as a function of a variable $u(R \rightarrow u), 2 n$ times and evaluates at $u=0$. That coefficient is the data that depends on the Mapping Torus while the integral over $X$ of the top form yields $c_{X}$. However, one can show that $I\left[\Sigma_{U}\right]$ obtained in this way is related to the Alexander polynomial of $I\left[\Sigma_{U}\right]$ (and its derivatives). This is a special example of the more general result that for all three manifolds with $b_{1}=1$, the partition function is a function of the Alexander polynomial of that manifold. This last fact is established in [7] using a path integral argument that is quite different to the one employed here.

There are some easy checks that one can make on (4.26). Firstly when $\Sigma_{g}=S^{2}$ the determinant is formally unity. This gives us back (3.20). When $\Sigma_{g}=T^{2}$, with $U=\mathbb{I}$, the determinant is

$$
\begin{aligned}
\operatorname{Det}\left(\mathbb{I} \otimes \mathbb{I}-\mathbb{I} \otimes \mathrm{e}^{R}\right)^{1 / 2} & =\operatorname{Det}\left(\mathbb{I}-\mathrm{e}^{R}\right) \\
& =-\prod 2 \sinh \left(x_{i} / 2\right),
\end{aligned}
$$

which means that

$$
-\mathrm{Todd}\left(T X_{\mathbb{C}}\right) \operatorname{Det}\left(\mathbb{I} \otimes \mathbb{I}-\mathbb{I} \otimes \mathrm{e}^{R}\right)^{1 / 2}=\prod x_{i}=\mathrm{e}(X) .
$$

\footnotetext{
${ }^{9}$ At one point in the derivation in [4] the fact that for a group valued field $\operatorname{det}(\mathrm{Adg})_{\mathbf{k}}=1$ is used. The equivalent, $\operatorname{det}\left(e^{R}\right)=1$, in the present context holds as $c_{1}(X)=0$, this being another point of contact with Chern-Simons theory.
} 
This reproduces the the result for $T^{2}$. Notice, however, that there is an ambiguity in the choice of the root of the determinant. The choice I have made is consistent with the formula, derived below, for the mapping torus. There the sign is set by demanding that it agrees with the formula for $S^{2} \times S^{1}$.

Recall that for a curve of genus greater than one the quantum mechanics path integral for $\Sigma_{g} \times S^{1}$ vanishes as one is obtaining too high powers of the curvature two form. In the present situation, we can see the same result for the same reason. From the general formula (4.26) one finds that with $U=\mathbb{I}$,

$$
\operatorname{Det}\left(\mathbb{I} \otimes \mathbb{I}-\mathbb{I} \otimes \mathrm{e}^{R}\right)^{1 / 2}=\operatorname{Det}\left(\mathbb{I}-\mathrm{e}^{R}\right)^{g}
$$

the determinant starts off as $\left(\prod x_{i}\right)^{g}$, which vanishes when $g \geq 2$.

\subsection{The Casson Invariant for Mapping-Tori}

When $\operatorname{dim}_{\mathbb{R}} X=4$, we are (essentially) calculating the Casson invariant. To begin with let the Riemann surface be a Torus. In such a situation the Mapping-Torus is a Torus bundle over the circle. The matrix $\mathrm{U}$, is now an element of $S L(2, \mathbb{Z})$ and takes the form

$$
U=\left(\begin{array}{ll}
p & q \\
r & s
\end{array}\right), \text { with } p s-q r=1, \text { and } p, q, r, s \in \mathbb{Z} .
$$

One calculates that

$$
\begin{aligned}
\operatorname{Det}\left(U \otimes \mathbb{I}-\mathbb{I} \otimes \mathrm{e}^{R}\right)^{1 / 2} & =\operatorname{Det}\left(\begin{array}{cc}
p \mathbb{I}-\mathrm{e}^{R} & q \mathbb{I} \\
r \mathbb{I} & s \mathbb{I}-\mathrm{e}^{R}
\end{array}\right)^{1 / 2} \\
& =\operatorname{Det}\left(\mathbb{I}-(p+s) \mathrm{e}^{R}+\mathrm{e}^{2 R}\right)^{1 / 2} \\
& =\operatorname{Det}(2 \cosh R-(p+s) \mathbb{I})^{1 / 2}
\end{aligned}
$$

When $\operatorname{dim}_{\mathbb{R}} X=4$ the (2-form) eigenvalues of $R$ are $x$ and $-x$ (since the manifold is Ricci flat). This means that in this case we have

$$
\operatorname{Det}(2 \cosh R-(p+s) \mathbb{I})^{1 / 2}=(2 \cosh x-(p+s)),
$$

and

$$
\begin{aligned}
Z_{X}^{R W}\left[T_{U}^{2}\right] & =\int_{X}-\frac{x^{2}}{4 \sinh ^{2} x / 2}(2 \cosh x-(p+s)) \\
& =-\frac{1}{2} \int_{X} x^{2} \cdot\left(\frac{u^{2}}{4 \sinh ^{2} u / 2}(2 \cosh u-(p+s))\right)_{u=0}^{\prime \prime} \\
& =c_{X} I\left[T_{U}^{2}\right]
\end{aligned}
$$


where

$$
\begin{aligned}
I\left[T_{U}^{2}\right] & =-\left(\frac{u^{2}}{4 \sinh ^{2} u / 2}(2 \cosh u-(p+s))\right)_{u=0}^{\prime \prime} \\
& =-\frac{1}{6}(p+s+10)
\end{aligned}
$$

and

$$
c_{X}=-\frac{1}{4} b_{\theta}(X),
$$

with

$$
b_{\theta}(X)=-2 \int_{X} x^{2} .
$$

All of this is in complete agreement with the calculations of Rozansky and Witten who arrive at these formula in two different (from the present derivation) ways. Their first method is to use the result from Chern-Simons theory that one is calculating the second derivative of the Ray-Singer torsion of the manifold while the second method is to make use of the explicit action of $U$ on the Hilbert space of states when $X$ is a K3 surface.

For hyper-Kähler manifolds of real dimension 4 and for $\Sigma_{g}$ with any genus $g$,

$$
\begin{aligned}
Z_{X}^{R W}\left[\Sigma_{U}\right] & =-\int_{X} \operatorname{Todd}\left(T X_{\mathbb{C}}\right) \operatorname{Det}(U-\mathbb{I})-\int_{X} \operatorname{Det}\left(U \otimes \mathbb{I}-\mathbb{I} \otimes \mathrm{e}^{R}\right)^{1 / 2} \\
& =c_{X}\left(\frac{1}{6}+\operatorname{Tr}(U-\mathbb{I})^{-1}+\operatorname{Tr}(U-\mathbb{I})^{-2}\right) \operatorname{Det}(U-\mathbb{I})
\end{aligned}
$$

\section{Reduction of the Rozansky-Witten Theory to Two Dimensions}

Given a theory on a $d+n$ dimensional manifold $Y_{d+n}$, there are two different ways to obtain a theory on a $d$ dimensional manifold $M_{d}$. The first is to consider a manifold of the form $Y_{d+n}=M_{d} \times \mathbb{R}^{n}$ and to simply 'forget' the dependence of the fields on the $\mathbb{R}^{n}$ coordinate-this goes by the name of dimensional reduction. The second is to consider the theory on a manifold $Y_{d+1}=M_{d} \times N_{n}$ and then to perform a harmonic eigenmode expansion of the fields with respect to some suitable operator on $N_{n}$ say, for example, in terms of eigenmodes of the Laplacian. The second approach is called Kaluza-Klein reduction, and yields a theory with a finite number of fields on 
$M_{d}$, when it is possible to integrate out most of the infinite tower of fields (one for each eigenmode). Typically, in conventional field theory, one takes the size of $N_{n}$ to be very small and then almost all the modes decouple. The error in doing this goes like the size of $N_{n}$, which for physical reasons (we do not see it) is very small. In a topological field theory one may have the freedom to vary the volume at will (since the theory should be metric independent) and therefore pass from the theory in $d+n$ dimensions to the theory in $d$, without error.

For the Rozansky-Witten theory on a three manifold of the form $\Sigma_{g} \times S^{1}$ there are then a number of approaches that one may take. One can, as in section 2.3, evaluate the path integral perturbatively by using the freedom to scale the coupling constant in front of the action which leads to the expressions already determined in terms of Greens functions on the three manifold. We have also used the Kaluza-Klein idea in order to equate the path integral of the Rozansky-Witten theory on $\Sigma_{g} \times S^{1}$ with a supersymmetric field theory on $S^{1}$. Alternatively one can, instead, shrink the radius of the $S^{1}$, that is one may perform a Kaluza-Klein reduction on the circle, to obtain an effective theory on $\Sigma_{g}$ that should also be equivalent to the Rozansky-Witten theory.

What theory will be obtained when one dimensionally reduces? There are two topological sigma models known in two dimensions that arise from the twist of the standard supersymmetric sigma model. They can be reduced to integrals over the moduli space of pseudo-holomorphic curves in the case of the $\mathbf{A}$-model or to an integral over constant maps in the case of the $\mathbf{B}$-model. A moments reflection will show that it must be the topological sigma model known as the B-model [23] that we obtain on reduction of the RozanskyWitten theory, since there too the path integral is reduced to an integral over constant maps. We can be more systematic about the relationship.

First the field content is the same. In a given complex structure of the hyper-Kähler manifold $X$, let $\phi^{I}$ be local holomorphic coordinates. The fields appearing in the $\mathbf{B}$-model are bosonic maps, $\phi^{i}$ from $\Sigma$ to $X$. There is also a Grassmann odd one form $\rho$ with values $\phi^{*}\left(\mathrm{~T}^{(1,0)} X\right)$ and two Grassmann odd zero forms with values in $\phi^{*}\left(\mathrm{~T}^{(0,1)} X\right)$. This is precisely the content of the Rozansky-Witten model on dimensional reduction. The bosonic field $\phi^{i}$ is clearly there. In the prefered complex structure one has $V=\phi^{*}\left(\mathrm{~T}^{(1,0)} X\right)$, so that $\eta$ is one of the Grassmann odd sections of $\phi^{*}\left(\mathrm{~T}^{(0,1)} X\right)$ while the component of $\chi$ in the $S^{1}$ direction provides the other. The one form is supplied by the rest of $\chi$. To make precise contact with the fields in [23] one makes use of the natural isomorphism between the holomorphic and 
anti-holomorphic tangent bundles of the hyper Kähler manifold $X$. One sets

$$
\eta^{\bar{I}}=g^{\bar{I} J} \epsilon_{J K} \eta^{K}, \quad \theta_{I}=\epsilon_{I J} \bar{\eta}^{J}, \quad \rho^{I}=\chi^{I} .
$$

Secondly one can compare the supersymmetry transformations, (2.11), after reduction directly with (4.2) in [23] and see, with the identifications made in (5.1), that they agree.

Of course, the ultimate test is that the actions agree as indeed they do. We can read off from $(3.1,3.2)$ the dimensionally reduced actions,

$$
\begin{aligned}
S_{1} & =\int_{\Sigma_{g}} \sqrt{h_{2}} \frac{1}{2} g_{i j} \partial_{\mu} \phi^{i} \partial^{\mu} \phi^{j}+\epsilon_{I J} \chi_{\mu}^{I} D^{\mu} \eta^{J} \\
S_{2} & =\int_{\Sigma_{g}}\left(\epsilon_{I J} \bar{\eta}^{I} D \chi^{J}+\Omega_{I J K L} \chi^{I} \chi^{J} \bar{\eta}^{K} \eta^{L}\right),
\end{aligned}
$$

which are in agreement with (4.3), (4.4) and (4.5) of [23].

There is, in principle, an anomaly in the B-model as the fields that make up the quadratic part of the Grassmann action are sections of different bundles. The fermionic determinant will thus have an anomaly unless certain conditions are met. Indeed it turns out that $X$ must satisfy $c_{1}(X)=0$. The fact that the manifold $X$ that appears in the Rozansky-Witten theory is hyper-Kähler ensures that the B-model makes sense.

We have not shown the equivalence of the generalized Casson invariant on a three manifold $M=\Sigma \times S^{1}$ with the $\mathbf{B}$-model, for the same target space $X$. To do that one would have to show that shrinking the $S^{1}$ reproduces the B-model. They are, infact, not equivalent; see footnote 11 for a brief discussion of this point.

\subsection{Observables}

As shown in [23] the observables of the B-model are naturally equivalent to,

$$
\oplus_{p, q} H^{(0, p)}\left(X, \bigwedge^{q} \mathrm{~T}^{(1,0)} X\right)
$$

As discussed by Witten [23] in order to describe the mirror map from the point of view of the topological sigma models one needs to thicken the moduli space a bit. One partial thickening is to define a $\mathbf{B}$ model which makes sense as one changes the complex structure of $X$. This is what Witten called a classical deformation. There are other thickenings that one may consider 
and one such example may be found in [23]. Fortunately, for us, the model of Rozansky and Witten comes equipped with a supersymmetry that does not require the specification of the complex structure of $X$. This property passes down to the $\mathbf{B}$-model of this section. Hence, for a hyper-Kähler target space the $\mathbf{B}$ model $(5.2,5.3)$ is well defined for all the complex structures that are compatible with the hyper-Kähler structure. The essence of the matter is that the action enjoys two linearly independent BRST symmetries the second BRST supersymmetry being,

$$
\begin{array}{ll}
Q \phi^{I}=\eta^{I}, & Q \phi^{\bar{I}}=0 \\
Q \eta^{I}=0, & Q \chi^{I}=\epsilon^{I J} g_{J} \bar{K} d \phi^{\bar{K}}-\Gamma_{J K}^{I} \eta^{J} \chi^{K}
\end{array}
$$

and, furthermore, it is exact with respect to both $Q$ and $\bar{Q} \cdot A_{\bar{I}}{ }^{J}$, the element in $H^{(0,1)}\left(X, T^{(1,0)} X\right)$ that deforms the complex structure while preserving the hyper-Kähler structure, is proportional to $g_{\bar{I} K} \epsilon^{K J}$.

One can now compare this with the proposal of Labastida and Marino [11] for perturbing the $\mathbf{B}$-model so that it incorporates complex structure deformations. From the discusion of the previous paragraph we should find that the perturbed B-model agrees with the unperturbed B-model. They proposed a deformed action together with a deformed BRST supersymmetry. That supersymmetry, their equation (26), is a linear combination of the $Q$ and $\bar{Q}$ supersymmetries and the action ${ }^{10}$, their equation (32), agrees with $(5.2,5.3)$.

\subsection{Reduction of the Kapranov-Kontsevich Theory to Two Dimensions}

There is a refinement of the Rozansky-Witten theory due to Kontsevich [10] and Kapranov [8]. The point is that one may lift the requirement that $X$ be hyper-Kähler. Instead one considers $X$ to be a complex manifold with a holomorphic symplectic structure. This means that $X$ comes equipped with a two form $\epsilon$ which is closed, $d \epsilon=0$, holomorphic (that is a $(2,0)$ form) and of maximal rank (non-degenerate), $\epsilon^{n} \neq 0$. Manifolds of this type also have vanishing $c_{1}(X)$. The corresponding three dimensional field theory, which requires a choice of Hermitian metric, is described in the appendix of [16].

The model that one obtains is actually a generalisation of the $\mathbf{B}$-model as holomorphic symplectic manifolds do not have to be Kähler and so, in

\footnotetext{
${ }^{10}$ To show the equivalence of the transformation rules and of the action one needs to remember that $D_{L}\left(g_{\bar{I} K} \epsilon^{K J}\right)=0$.
} 
particular, do not have to be Calabi-Yau manifolds. Recall that the topological models are obtained by twisting the $N=2$ sigma model. The extended supersymmetric theory is formulated on a Kähler manifold and so by construction the $\mathbf{A}$ and $\mathbf{B}$ models are defined on Kähler manifolds. However, the $\mathbf{A}$-model makes sense without the Kähler condition on $X$, it is enough that $X$ admit an almost complex structure. Consistency for the $\mathbf{B}$ model requires that $c_{1}(X)=0$.

The action for the $\mathbf{B}$-model on a Riemann surface $\Sigma_{g}$, is found just by dimensional reduction ${ }^{11}$ of the model presented in [16] and setting $\chi_{t}^{I}=\bar{\eta}^{I}$ where $t$ is the $S^{1}$ co-ordinate,

$$
S_{\mathbf{B}}=c_{1} \int_{\Sigma} L_{1}+c_{2} \int_{\Sigma} L_{2}
$$

where

$$
L_{1}=\left\{Q, g_{I} \chi_{\mu}^{I} \partial^{\mu} \bar{\phi}^{\bar{I}}\right\}
$$

and

$$
\begin{aligned}
L_{2}= & \epsilon_{I J} \chi^{I} D \bar{\eta}^{J}-\frac{1}{3} \epsilon_{I J} R_{K L}^{J} \bar{M}^{I} \chi^{K} \bar{\eta}^{L} \eta^{\bar{M}}-\frac{1}{6} \epsilon_{I J} R_{K L \bar{M}}^{J} \bar{\eta}^{I} \chi^{K} \chi^{L} \eta^{\bar{M}} \\
& +\frac{1}{6}\left(\nabla_{L} \epsilon_{I K}\right) d \phi^{I}\left(\chi^{K} \bar{\eta}^{L}+\chi^{L} \bar{\eta}^{K}\right) .
\end{aligned}
$$

The BRST operator $Q$ acts in the following way

$$
\begin{array}{lll}
Q \phi^{I}=0, & Q \phi^{\bar{I}}=\eta^{\bar{I}} & Q \bar{\eta}^{I}=0 \\
Q \eta^{\bar{I}}=0, & Q \chi^{I}=-d \phi^{I} . &
\end{array}
$$

It was also pointed out in [16] that the BRST class of $L_{2}$ is independent of the connection $\Gamma_{J K}^{I}$, that is, one can add to $\Gamma_{J K}^{I}$ a tensor $A_{J K}^{I}$, and $L_{2}$ is then changed by a BRST exact term. One immediate implication of this is that it is BRST equivalent to work with a covariantly constant $\epsilon$. We have to show that even though

$$
\nabla_{K}(\Gamma) \epsilon_{I J}=\nabla_{K} \epsilon_{I J} \neq 0
$$

one can choose a new connection $\nabla_{K}(\Gamma+A)$ so that

$$
\nabla_{K}(\Gamma+A) \epsilon_{I J}=\nabla_{K}^{\prime} \epsilon_{I J}=0 .
$$

\footnotetext{
${ }^{11}$ Since the topological theory should not depend on the metric one puts on the three manifold one could also consider the theory in three dimensions and take the radius of the $S^{1}$ to zero. This will not give back the two dimensional model. Rather one will obtain the two dimensional theory together with the insertion of an operator. It is the expectation value of this operator that will give back the Casson invariant.
} 
Let $\hat{\epsilon}^{I J}$ be the matrix that inverts $\epsilon_{I J}$,

$$
\hat{\epsilon}^{I J} \epsilon_{J K}=\delta_{K}^{I}
$$

Such a matrix exits as $\epsilon$ is non-degenerate, furthermore it is holomorphic since $\epsilon$ is. The required tensor $A_{J K}^{I}$ is

$$
A_{J K}^{I}=\frac{1}{3} \hat{\epsilon}^{I M}\left(\nabla_{J} \epsilon_{M K}+\nabla_{K} \epsilon_{M J}\right) .
$$

One finds,

$$
\begin{aligned}
\nabla_{K}^{\prime} \epsilon_{I J} & =\nabla_{K} \epsilon_{I J}-A_{K I}^{M} \epsilon_{M J}-A_{K J}^{M} \epsilon_{I M} \\
& =\nabla_{K} \epsilon_{I J}+\frac{1}{3}\left(\nabla_{K} \epsilon_{J I}+\nabla_{I} \epsilon_{J K}\right)-\frac{1}{3}\left(\nabla_{K} \epsilon_{I J}+\nabla_{J} \epsilon_{I K}\right) \\
& =\frac{1}{3}\left(\nabla_{K} \epsilon_{I J}+\nabla_{I} \epsilon_{J K}+\nabla_{J} \epsilon_{K I}\right) \\
& =\frac{1}{3}\left(\partial_{K} \epsilon_{I J}+\partial_{I} \epsilon_{J K}+\partial_{J} \epsilon_{K I}\right) \\
& =0
\end{aligned}
$$

and the last equality follows since $\epsilon$ is also closed.

Consequently, we see that there is precisely enough information in having a holomorphic symplectic structure to be able to find a connection with respect to which $\epsilon$ is covariantly constant. BRST invariance tells us that we may work with such a connection without changing the results of the topological field theory. As far as the theory is concerned this means that we can just as well drop the last line of (5.8), providing that we understand all covariant derivatives to be with respect to the new connection. Once one has chosen the connection so that the holomorphic symplectic structure $\epsilon$ is also parallel then all the Chern forms ${ }^{12}$ of $c_{2 j+1}(X)$ vanish [9]. Some stringent conditions on the cohomology of $X$ can be deduced from these facts, [9].

Returning to the properties of the field theory, the scaling arguments used to deduce that the Rozansky-Witten model devolves to an integral over constant maps can be directly taken over to the Kapranov-Kontsevich theory. The new B-model (5.6), likewise can be shown to devolve to an integral on the space of constant maps, that is to an integral over $X$.

This generalised B-model is probably obtained from the twisting of an $N=2$ sigma model with non-zero $B$ field. In particular, one expects that $B=\epsilon$.

\footnotetext{
${ }^{12}$ Meaning that it is not only the $(2 j+1)$ th Chern numbers that vanish, but the ChernWeil representatives themselves.
} 


\section{Acknowledgements}

I would like to thank N. Habegger and M. Narasimhan and especially M. Blau for discussions on this work.

\section{References}

[1] L. Alvarez-Gaume, Supersymmetry and the Atiyah-Singer Index Theorem, Commun. Math. Phys. 90, 161-173 (1983).

[2] M. Blau and G. Thompson, N=2 Topological Gauge Theory, the Euler Characteristic of Moduli Spaces and the Casson Invariant, Commun. Math. Phys. 152, 41-72 (1993), hep-th/9112012.

[3] M. Blau and G. Thompson, Euclidean SYM Theories by Time Reduction and Special Holonomy Manifolds, Phys. Lett. B415, 242-252 (1997), hepth/9706225.

[4] M. Blau and G. Thompson, Derivation of the Verlinde Formula from ChernSimons Theory and the G/G Model, Nucl. Phys. B408, 345-390 (1993), hepth/9305010.

[5] H. Boden and C. Herald, The SU(3) Casson Invariant for Integral Homology 3-Spheres, math.DG/9809124.

[6] A. Casson, Lectures at the MSRI, (1985).

[7] N. Habegger and G. Thompson, The Universal Perturbative Quantum 3Manifold Invariant, in preperation.

[8] M. Kapranov, Rozansky-Witten Invariants via Atiyah Classes, alggeom $/ 9704009$.

[9] S. Kobayashi, Differential Geometry of Complex Vector Bundles, Princeton University Press, Princeton, (1987).

[10] M. Kontsevich, Rozansky-Witten Invariants via Formal Geometry, dgga/ 9704009 .

[11] J. Labastida and M. Mirano, Type B Topological Matter, Kodaira-Spencer theory and Mirror Symmetry, Phys. Lett. B333 386-395 (1994), hep-th/9405151.

[12] C. Lescop, Global Surgery Formula for the Casson-Walker Invariant, Annals of Mathematics Studies, 140, Princeton University Press, (1996).

[13] T.T.Q. Le, J. Murakami, T. Ohtsuki, On a universal perturbative invariant of 3-manifolds, Topology 37, 539-574 (1998).

[14] M. Marcoli, Seiberg-Witten Gauge Theory, to appear in Text and Readings in Mathematics, Editor T. Ramadas, Hindustan Book Agency, New Delhi.

[15] G. Moore and E. Witten, Integration over the u-plane in Donaldson Theory, hep-th/9709193. 
[16] L. Rozansky and E. Witten, Hyper-Kähler Geometry and Invariants of Three Manifolds, hep-th/9612216.

[17] N. Seiberg and E. Witten, Monopoles, Duality and Chiral Symmetry Breaking in N=2 Supersymmetric QCD, Nucl. Phys. B431, 484-550 (1994), hepth/ 9408099 .

[18] N. Seiberg and E. Witten, Gauge Dynamics and Compactification to Three Dimensions, hep-th/9607163.

[19] C. Taubes, Casson's Invariant and Gauge Theory, J. Diff. Geom. 31, 547 (1990).

[20] K. Walker, An Extension of Casson's Invariant, Annals of Mathematics Studies, 126, Princeton University Press, (1992).

[21] E. Witten, Topology Changing Amplitudes in $2+1$ dimensional Gravity, Nucl. Phys. B323, 281 (1990).

[22] E. Witten, Supersymmetry and Morse Theory, J. Diff. Geom. 17, 661 (1982).

[23] E. Witten, Mirror Manifolds and Topological Field Theory, Essays on Mirror Manifolds, S-T Yau editor, International Press, (1992). 